\title{
Interstellar extinction in the open clusters towards galactic longitude around $130^{\circ}$
}

\author{
A. K. Pandey ${ }^{1}$, K. Upadhyay ${ }^{1}$, Y. Nakada ${ }^{2}$, and K. Ogura ${ }^{3}$ \\ 1 State Observatory, Manora Peak, Naini Tal, 263 129, Uttaranchal, India \\ 2 Kiso Observatory, School of Science, University of Tokyo, Mitake-mura, Kiso-gun, Nagano 397-0101, Japan \\ 3 Kokugakuin University, Higashi, Shibuya-ku, Tokyo 150-8440, Japan
}

Received 26 March 2002 / Accepted 5 September 2002

\begin{abstract}
In this paper we present a detailed study of the intra-cluster reddening material in the young open clusters located around $l \sim 130^{\circ}$ using colour-excess diagrams and two-colour diagrams. The study supports the universality of the extinction curves for $\lambda \geq \lambda_{J}$, whereas for shorter wavelengths the curve depends upon the value of the $R_{\text {cluster }}$ (total-to-selective absorption in the cluster region). The value of $R_{\text {cluster }}$ in the case of NGC 654, NGC 869 and NGC 884 is found to be normal, whereas the value of $R_{\text {cluster }}$ in the cluster regions NGC 1502 and IC 1805 indicates an anomalous reddening law in these regions. In the case of NGC 1502 the value of $R_{\text {cluster }}$ is found to be lower $(2.57 \pm 0.27)$ whereas in the case of IC 1805 it is higher (3.56 \pm 0.29$)$ than the normal value of 3.1. Although the intra-cluster material indicates a higher value of $R_{\text {cluster }}$ in the NGC 663 region, the error in the estimation of $R_{\text {cluster }}$ is too large to conclude anything. It is also found that the extinction process in the $U$ band in the case of NGC 663 seems to be less efficient, whereas in the case of NGC 869 the process is more efficient.
\end{abstract}

Key words. ISM: dust, extinction - ISM: general - open clusters and associations: general

\section{Introduction}

Photometric studies are one of the most valuable and efficient tools for determining the physical properties of open clusters (e.g. distance, age, etc.) and the interstellar matter (ISM) within the cluster as well as along the line of sight of the cluster. The accurate determination of the distance of star clusters is crucial for a wide range of astronomical studies. The interstellar extinction and the ratio of the total-to-selective extinction $R=A_{V} / E(B-V)$ towards the cluster are important quantities that must be accurately known to determine the distances photometrically.

Observations of star clusters in the near-infrared bands, especially of young clusters which are still embedded in remnants of their parental clouds, are very useful to study the extinction behaviour in these clusters. Observations of interstellar extinction due to the intra-cluster matter yield fundamental information on the optical properties of the particles responsible for extinction. The analysis of extinction curves in $20 \mathrm{OB}$ stellar associations indicates that big complexes are obscured by the same type of interstellar matter (Kiszkurno et al. 1984). On the other hand Krełowski \& Ströbel (1983) compared the extinction law in two rich stellar aggregates, namely Per OB1 and Sco OB2, situated in different parts of the galactic disk. They found that the average extinction curves of the two aggregates

Send offprint requests to: A. K. Pandey,

e-mail: pandey@upso.ernet.in seem to differ substantially. The differences are large in the farUV, which leads to the conclusion that an average extinction law cannot be applied to all associations. In an analysis of two different complexes of young stars (Sco-Ori region and Perseus region) Krełowski \& Ströbel (1987) found different shapes of extinction curves, supporting the assumption that the obscuring material in these two young star complexes have different physical properties. Consequently, in the case of young open clusters, the average extinction curve should not be used to correct spectral or photometric data for interstellar extinction.

There is much evidence in the literature that indicates significant variations in the properties of the interstellar extinction and these refer mainly to high values of the total-to-selective extinction ratio $R$ as compared to the normal value, 3.1, for the galactic diffuse medium (see e.g. Pandey et al. 2000 and references therein). Whittet (1977) reported that the value of $R$ in the galactic plane can be represented by a sinusoidal function of the form $R=3.08+0.17 \sin \left(l+175^{\circ}\right)$, which indicates a minimum value of $R$ at $l \approx 95^{\circ}$. However, Tapia et al. (1991) found a value of $R=2.42 \pm 0.09$ for the cluster NGC $1502\left(l=143.7^{\circ}\right)$, which is significantly lower than the average galactic value of 3.1.

The above discussions indicate that the interstellar extinction shows a large range of variability from one line of sight to another. A precise knowledge of the spatial variability of interstellar extinction is important for the following reasons;

(i) Since the extinction depends on the optical properties of the dust grains, it can reveal information about the 
Table 1. The details of the clusters used in the study.

\begin{tabular}{llrllll}
\hline \hline Cluster & $l$ & \multicolumn{1}{c}{$b$} & $\begin{array}{l}\text { Distance } \\
(\mathrm{pc})\end{array}$ & $\begin{array}{l}\text { Log age } \\
(\mathrm{Yr})\end{array}$ & $E(B-V)$ & $\begin{array}{l}\text { Available } \\
\text { photometric data }\end{array}$ \\
\hline IC 1590 & 123.13 & -06.24 & 2940 & 6.54 & 0.32 & $U B V I_{\mathrm{c}} J H K$ \\
Be 62 & 123.99 & 01.10 & 2513 & 7.22 & 0.85 & $U B V$ \\
NGC 436 & 126.07 & -03.91 & 2942 & 7.78 & 0.48 & $U B V I_{\mathrm{c}}$ \\
NGC 457 & 126.56 & -04.35 & 2796 & 7.15 & 0.48 & $U B V I_{\mathrm{c}}$ \\
NGC 581 & 128.02 & -01.76 & 2241 & 7.13 & 0.44 & $U B V I_{\mathrm{c}}$ \\
Tr 1 & 128.22 & -01.14 & 2520 & 7.43 & 0.63 & $U B V$ \\
NGC 637 & 128.55 & 01.70 & 2372 & 6.96 & 0.70 & $U B V$ \\
NGC 654 & 129.09 & -00.35 & 2422 & 7.08 & 0.85 & $U B V R_{\mathrm{c}} I_{\mathrm{c}}$ \\
NGC 663 & 129.46 & -00.94 & 2284 & 7.13 & 0.82 & $U B V I_{\mathrm{c}} J H K$ \\
Be 7 & 130.13 & 00.37 & 2570 & 6.60 & 0.80 & $U B V$ \\
NGC 869 & 134.63 & -03.72 & 2115 & 7.10 & 0.58 & $U B V R_{\mathrm{c}} I_{\mathrm{c}} J H K$ \\
IC 1805 & 134.74 & 00.92 & 2195 & 6.67 & 0.80 & $U B V R_{\mathrm{c}} I_{\mathrm{c}} J H K$ \\
NGC 884 & 135.08 & -03.60 & 2487 & 7.15 & 0.58 & $U B V R_{\mathrm{c}} I_{\mathrm{c}} J H K$ \\
NGC 1502 & 143.65 & 07.62 & 900 & 7.03 & 0.74 & $U B V I_{\mathrm{c}} J H K$ \\
\hline
\end{tabular}

composition and size distribution of the grains. Consequently, variation of the extinction from one direction to other may reveal the degree and nature of dust grain processing in the ISM (Fitzpatrick 1999).

(ii) Since astronomical objects are viewed through interstellar dust, the wavelength dependence of extinction is required to remove the effects of dust obscuration from observed energy distributions.

Uncertainties of extinction estimates limit the accuracy of dereddened energy distributions. Such uncertainties might be acceptably small for very lightly reddened objects but can become important for modestly reddened objects. The intracluster extinction due to the remains of the star-forming molecular cloud decreases systematically with the age of the cluster (cf. Pandey et al. 1990).

While analysing the $U B V I_{\mathrm{c}}$ data of NGC 663 we found that the $V /(U-B)$ colour-magnitude diagram (CMD) for NGC 663 cannot be explained by a normal value of the extinction ratio $E(U-B) / E(B-V)=0.72$ (see Sect. 3). Some clusters in the direction of NGC 663 also appear to show abnormal extinction properties. This motivated us to study the interstellar extinction in detail towards the direction of the cluster NGC 663.

\section{Data}

The online available database at http : //obswww . unige . ch/ webda/ by Mermilliod (1995) provides an excellent compilation of the data on open clusters. We selected a range of $120^{\circ}<l<150^{\circ}$ to study the intra-cluster extinction in open clusters. The online database lists 103 clusters in this longitude range. The parameters, such as distance, $E(B-V)$, age etc., are available for only 54 clusters. Seventeen clusters in this direction are quite young (log age $<7.5)$ and are suitable to study the behaviour of intra-cluster ISM. $U B V$ CCD photometry for nine young clusters of the above sample is given by Phelps \& Janes (1994). A casual look at the $(U-B) /(B-V)$ two-colour diagrams (TCDs) given by Phelps \& Janes (1994, hereafter PJ94) indicates that the photometric data of 3 clusters, namely Be 62,
NGC 637 and NGC 663, do not satisfy the main-sequence (MS) curve, where apparent $U V$ excess can be seen for stars having spectral type later than $A$. PJ1994 concluded that this may be due to $U V$ excess of pre-main-sequence (PMS) stars and this part of the $(U-B) /(B-V)$ TCD is not suitable for reddening estimation. We will discuss $(U-B) /(B-V)$ TCDs in ensuing sections. The present analysis has been carried out using photometric data of 14 clusters and details are given Table 1.

\section{Extinction}

One of the main characterstics of the diffuse interstellar matter in the Galaxy is its irregular structure. This makes it difficult to map the extinction as a function of galactic longitude and distance. Young open clusters also show differential reddening due to the remains of the associated parental clouds. The differential reddening decreases with the age of the clusters (cf. Pandey et al. 1990). However, it does not show any correlation with the location of the cluster in the galactic disk.

Thus the extinction in star clusters arises due to two distinct sources; (i) the general interstellar medium (ISM) in the foreground of the cluster, and (ii) the localized cloud associated with the cluster. While for the former component a value of $R=3.1$ is well accepted (Wegner 1993; Lida et al. 1995; Winkler 1997), for the intra-cluster regions the value of $R$ varies from 2.42 (Tapia et al. 1991) to 4.9 (Pandey et al. 2000 and references therein) or even higher depending upon the conditions occurring in the region.

\subsection{Extinction curve}

Extinction has often been analyzed using a two-colour normalizations of the form $E(\lambda-V) / E(B-V)$. In the present work the following methods are used to derive the ratio of $E(\lambda-V) / E(B-V)$.

a) Colour excess diagrams (CEDs); method "A"

The colour excesses of the stars in the cluster region can be obtained by comparing the observed colours of the stars with 

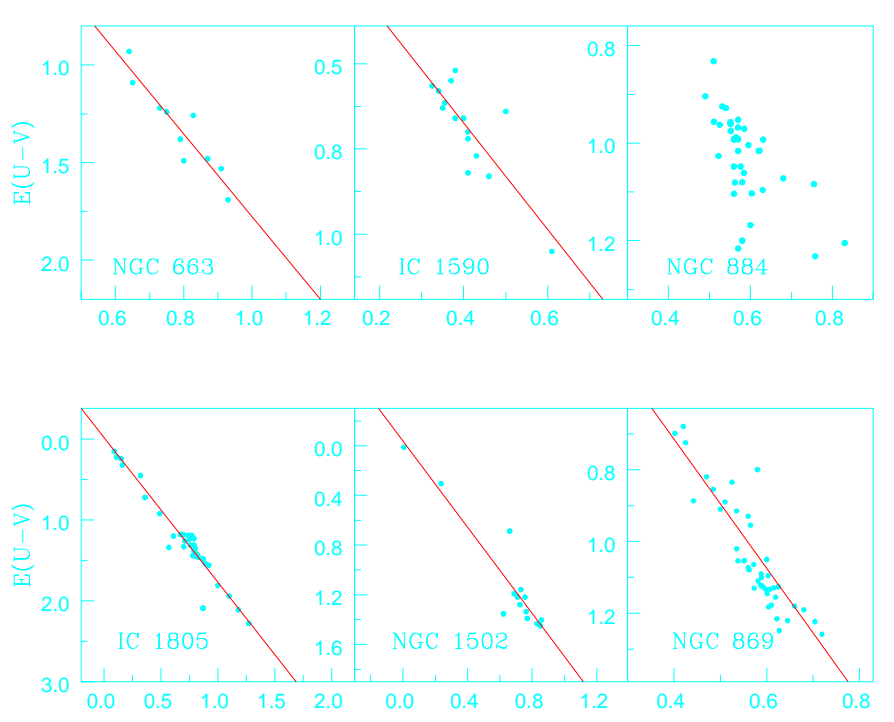

Fig. 1. $E(U-V)$ vs. $E(B-V)$ colour-excess diagrams. Straight line shows a least-square fit to the data.

their intrinsic colours derived from the MKK bi-dimensional spectral classification. For this purpose we used the data available in the online catalogue by Mermilliod (1995). When multiple data points are available for a star, we used those selected by Mermilliod and given as MKS in the online catalogue. The colour excess in a colour index $(\lambda-V)$ is obtained from the relation $E(\lambda-V)=(\lambda-V)-(\lambda-V)_{0}$, where $(\lambda-V)_{0}$ is the intrinsic colour index and $\lambda$ represents the magnitude in the $U B V R I J H K$ pass bands. Intrinsic colours are obtained from the MKK spectral type-luminosity class relation given by Schmidt-Kaler (1982) for $U B V$, by Johnson (1966) for $V R I$ converted to the Cousin's system using the relation given by Bessel (1979), and by Koornneef (1983) for VJHK.

The colour excesses $E(U-V), E(I-V), E(J-V), E(H-V)$ and $E(K-V)$ are shown as a function of $E(B-V)$ in Figs. 1, 2, 3 and 4 . The stars having $\mathrm{H}_{\alpha}$ emission features and stars apparently lying away from the general distribution are not included in the analysis. A least-squares fit to the data is shown by a straight line which gives the ratio of $E(\lambda-V) / E(B-V)$ for the stars in the cluster region. The slope of the line representing the ratio $E(\lambda-V) / E(B-V)$ along with the error is given in Table 2. In general, the least-square errors are quite large. The reason for the large errors is mainly the small sample and the small range in the $E(B-V)$. For comparison the colour excess ratios for the normal reddening law (cf. Johnson 1968; Dean et al. 1978) are also given in Table 2.

b) Two colour diagrams (TCDs); method "B"

In most of the cases the MKK spectral classification is available only for a few stars of the cluster, which make the CEDs quite noisy (see e.g. the CEDs of NGC 654, NGC 663, NGC 869 and NGC 884). The TCDs of the form of $(\lambda-V)$ vs. $(B-V)$, where $\lambda$ is one of the wavelength of the broad band filters $(R, I, J, H, K, L)$, provide an effective method for separating the influence of the normal extinction produced by the diffuse interstellar medium from that of the abnormal

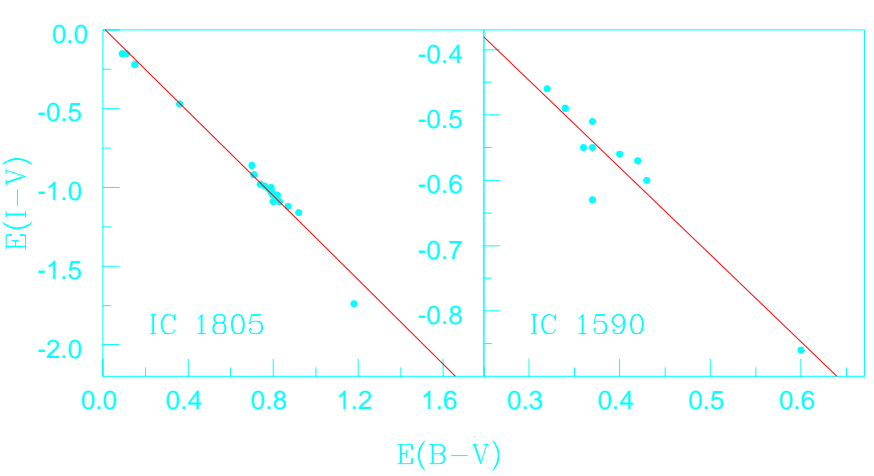

Fig. 2. $E(I-V)$ vs. $E(B-V)$ colour-excess diagrams.

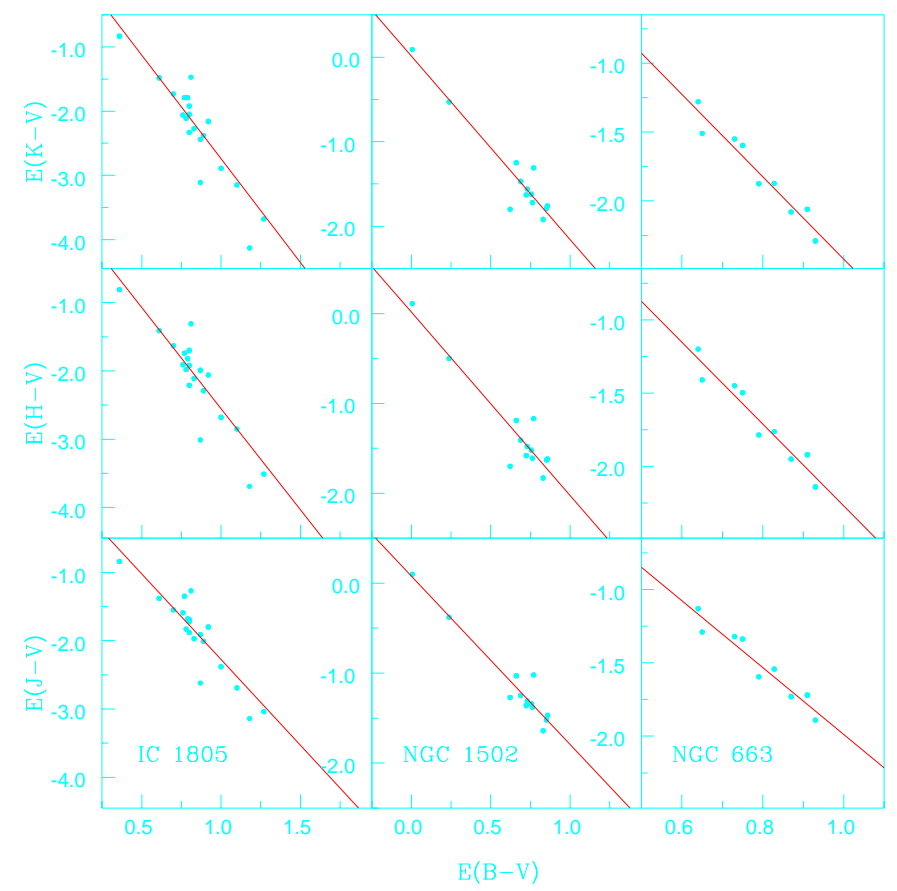

Fig. 3. $E(J-V), E(H-V), E(K-V)$ vs. $E(B-V)$ colour-excess diagrams for the clusters IC 1805, NGC 1502 and NGC 663.

extinction arising within regions having a peculiar distribution of dust sizes (cf. Chini \& Wargau 1990; Pandey et al. 2000). On these diagrams the unreddened MS and the normal reddening vector are practically parallel. This makes these diagrams useless for determining the amount of reddening, but instead, very useful for detecting anomalies in the reddening law. Chini \& Wargau (1990) and Pandey et al. (2000) used TCDs to study the anomalous extinction law in the clusters M 16 and NGC 3603 respectively. Figures 5, 6, 7 and 8 show TCDs for the central region of the clusters. We used the data of the central region of the clusters to reduce the contamination due to field stars. The stars apparently lying away from the general distribution are not included in the analysis. The slopes of the distribution, $m_{\text {cluster }}$, are given in Table 3. The slopes of the theoretical MS, $m_{\text {normal }}$, on the TCDs, obtained for the stellar models by Bertelli et al. (1994) are also given in Table 3. The errors associated with the slopes are significantly smaller than the errors obtained in the CEDs. The values of the $(\lambda-V) /(B-V)$ can be converted to 
Table 2. The colour excess ratios $E(\lambda-V) / E(B-V)$ obtained from the CEDs.

\begin{tabular}{llllll}
\hline \hline Cluster & $\frac{E(U-V)}{E(B-V)}$ & $\frac{E(I-V)}{E(B-V)}$ & $\frac{E(J-V)}{E(B-V)}$ & $\frac{E(H-V)}{E(B-V)}$ & $\frac{E(K-V)}{E(B-V)}$ \\
\hline NGC 654 & & & $-2.20 \pm 0.26$ & $-2.47 \pm 0.26$ & $-2.71 \pm 0.27$ \\
NGC 663 & $2.12 \pm 0.32$ & & $-2.28 \pm 0.25$ & $-2.79 \pm 0.30$ & $-2.99 \pm 0.30$ \\
NGC 869 & $1.90 \pm 0.13$ & & $-1.20 \pm 0.46$ & $-1.75 \pm 0.48$ & $-1.59 \pm 0.50$ \\
NGC 1502 & $1.74 \pm 0.18$ & & $-1.87 \pm 0.16$ & $-2.04 \pm 0.23$ & $-2.17 \pm 0.22$ \\
IC 1805 & $1.76 \pm 0.08$ & $-1.34 \pm 0.04$ & $-2.74 \pm 0.30$ & $-3.30 \pm 0.37$ & $-3.66 \pm 0.38$ \\
IC 1590 & $1.56 \pm 0.30$ & $-1.33 \pm 0.17$ & & & \\
Normal & 1.72 & -1.25 & -2.30 & -2.58 & -2.78 \\
\hline
\end{tabular}

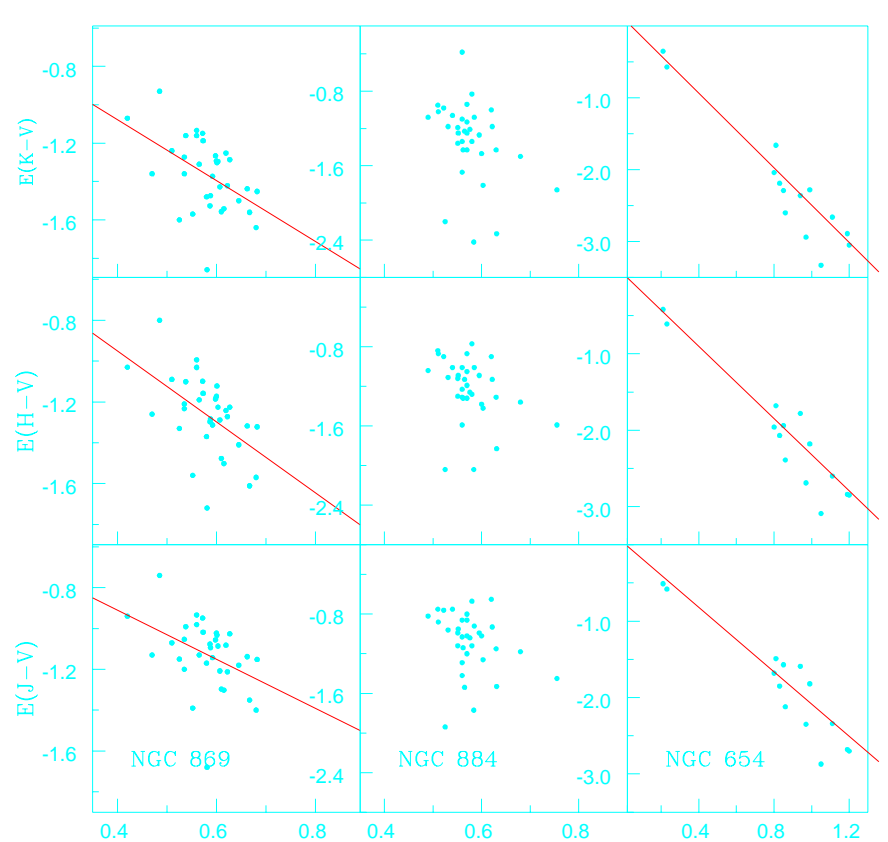

Fig. 4. Same as Fig. 3 but for the clusters NGC 869, NGC 884 and NGC 654

the ratio $E(\lambda-V) / E(B-V)$ using the following approximate relation;

$\frac{E(\lambda-V)}{E(B-V)}=\frac{m_{\text {cluster }}}{m_{\text {normal }}} \times\left[\frac{E(\lambda-V)}{E(B-V)}\right]_{\text {normal }}$.

c) $(U-B) /(B-V)$ two-colour diagram and colour-magnitude diagrams; method "C"

In absence of spectroscopic observations, the $(U-B) /(B-$ $V)$ TCD and colour-magnitude diagrams (CMDs) are important tools to study the interstellar reddening towards the cluster as well as intra-cluster reddening.

In the case of $(U-B) /(B-V)$ TCD a MS curve (e.g. given by Schmidt-Kaler 1982) is shifted along a reddening vector given by the ratio of $E(U-B) / E(B-V) \equiv X$ until a match between the MS and stellar distribution is found. In this method the reddening vector $X$ plays an important role. Over the years the observational as well as theoretical studies have used (or "misused", according to Turner 1994) a universal form for the mean galactic reddening law. However, theoretical as well as observational estimates for the reddening vector $X$ show a range from 0.62 to 0.80 (cf. Turner 1994). Recently

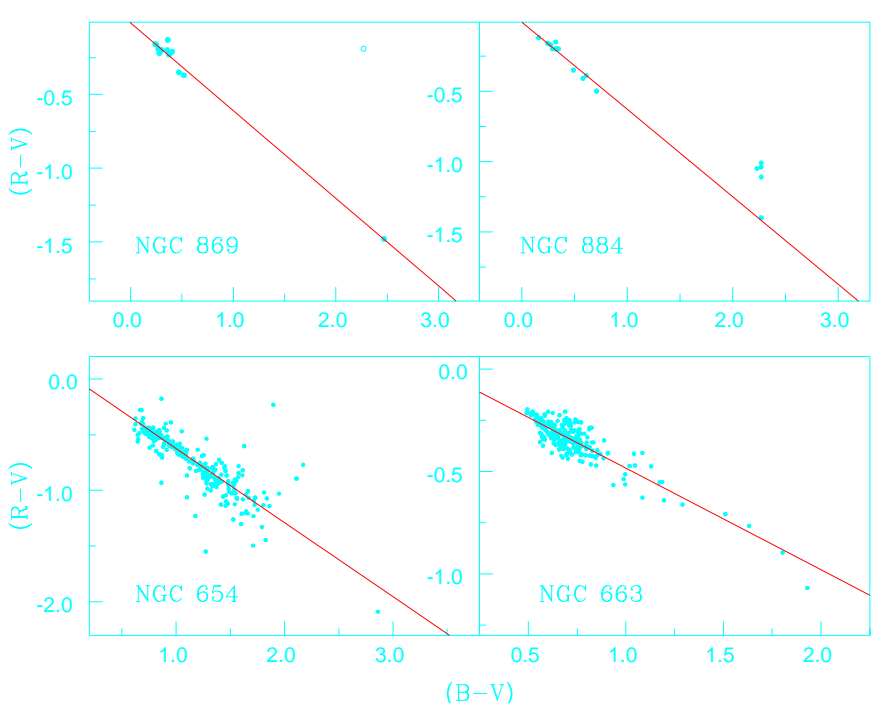

Fig. 5. $(R-V)$ vs. $(B-V)$ two-colour diagram. The data point shown by open circles is not included in the least-squares fit (see text).
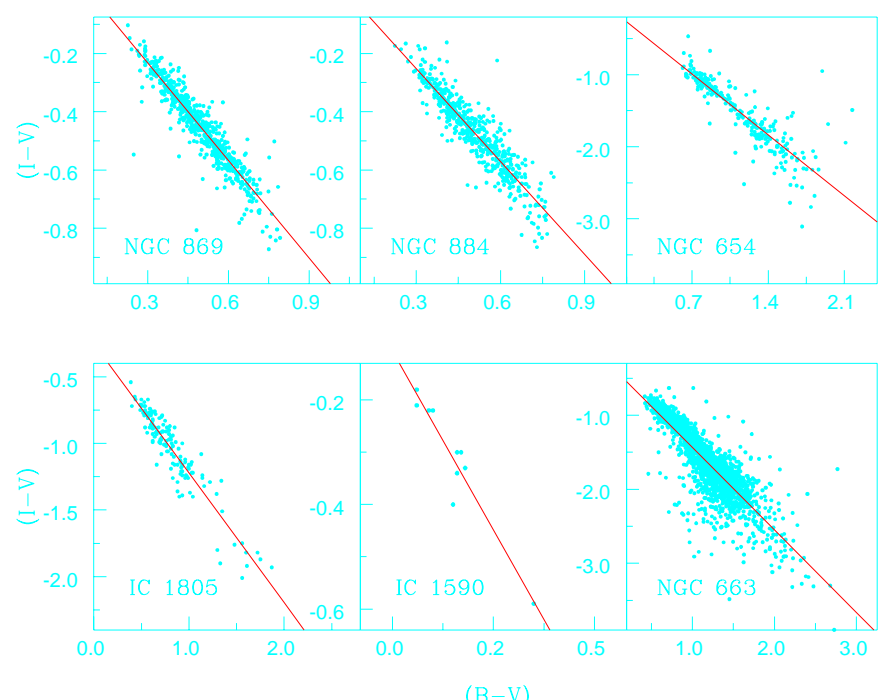

Fig. 6. $(I-V)$ vs. $(B-V)$ two-colour diagram.

DeGioia Eastwood et al. (2001) also preferred a value of $X=0.64$ in the case of $\operatorname{Tr} 14$. The variability of $X$ indicates the variations in the properties of the dust grains responsible for the extinction. High values of $X$ imply a dominance by dust grains of small cross sections while the small values of $X$ indicate a dominance of dust grains of larger cross section 
Table 3. The slopes of the distribution of stars obtained from the $(\lambda-V) /(B-V)$ TCDs.

\begin{tabular}{llllll}
\hline \hline Cluster & $\frac{(R-V)}{(B-V)}$ & $\frac{(I-V)}{(B-V)}$ & $\frac{(J-V)}{(B-V)}$ & $\frac{(H-V)}{(B-V)}$ & $\frac{(K-V)}{(B-V)}$ \\
\hline NGC 654 & $-0.61 \pm 0.02$ & $-1.20 \pm 0.04$ & $-2.16 \pm 0.25$ & $-2.39 \pm 0.23$ & $-2.50 \pm 0.25$ \\
NGC 663 & $-0.50 \pm 0.02$ & $-1.13 \pm 0.03$ & $-2.33 \pm 0.30$ & $-2.73 \pm 0.35$ & $-3.07 \pm 0.43$ \\
NGC 869 & $-0.60 \pm 0.02$ & $-1.12 \pm 0.02$ & $-1.90 \pm 0.10$ & $-2.41 \pm 0.10$ & $-2.53 \pm 0.11$ \\
NGC 884 & $-0.62 \pm 0.02$ & $-1.06 \pm 0.02$ & $-1.96 \pm 0.08$ & $-2.44 \pm 0.08$ & $-2.59 \pm 0.10$ \\
NGC 1502 & & & $-1.92 \pm 0.24$ & $-2.04 \pm 0.24$ & $-2.08 \pm 0.24$ \\
IC 1590 & & $-1.36 \pm 0.09$ & & & \\
IC 1805 & $-0.98 \pm 0.04$ & $-2.12 \pm 0.15$ & $-2.58 \pm 0.17$ & $-2.73 \pm 0.18$ \\
Normal & -0.55 & -1.1 & -1.96 & -2.42 & -2.60 \\
\hline
\end{tabular}

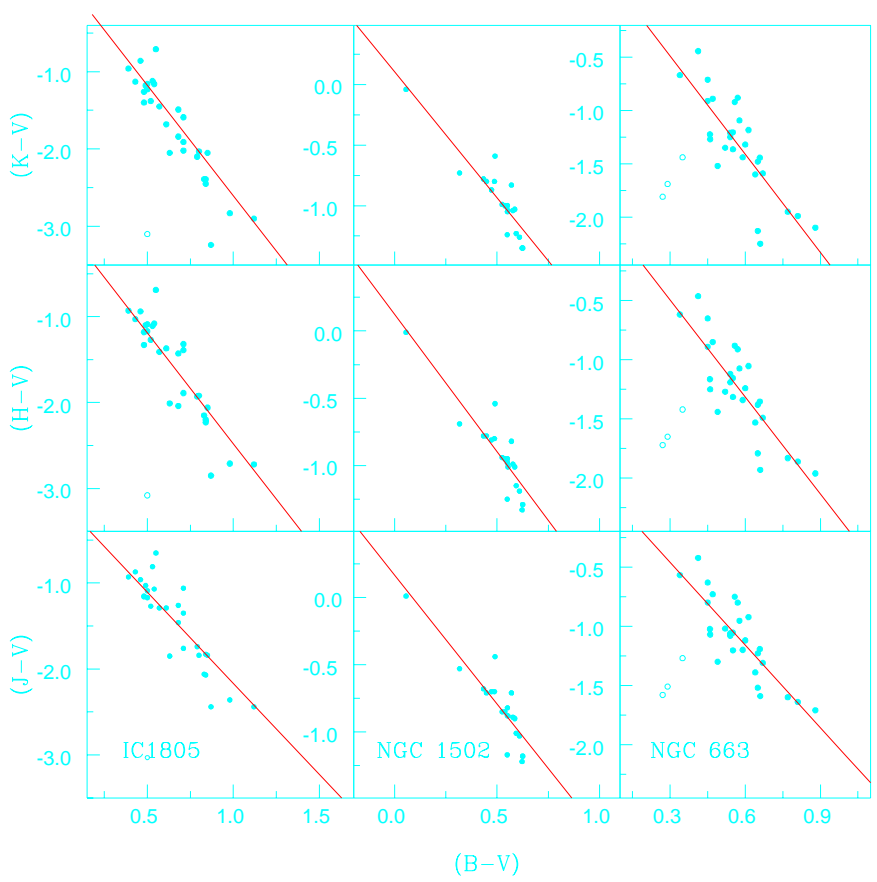

Fig. 7. $(J-V),(H-V),(K-V)$ vs. $(B-V)$ two-colour diagrams for the clusters IC 1805, NGC 1502 and NGC 663. The data point shown by open circles are not included in the least-squares fit (see text).

(cf. Turner 1994). Turner (1994) raised a question about the so called reddening-free parameter $Q[=(U-B)-0.72(B-V)]$; how can $Q$ be reddening-free when the reddening vector $X$ is different from 0.72 ?

As we have mentioned earlier, the distribution of stars in the $V /(U-B) \mathrm{CMD}$ of NGC 663 cannot be explained by a normal value of $X$ (i.e. 0.72$)$. The $(U-B) /(B-V)$ TCD and the $V /(B-V), V /(U-B) C M D s$ for the central region of the cluster NGC 663 are shown in Figs. 9 and 10 respectively. The data have been taken using the $105 \mathrm{~cm}$ Schmidt telescope of the Kiso Observatory, Japan (for details see Pandey et al. 2002). In Fig. 9, where the dashed curve shows the intrinsic MS by Schmidt-Kaler (1982) shifted along $X=0.72$, we find a disagreement between the observations and the MS at $(B-V) \sim 0.90$. PJ94 explained the disagreement due to the presence of pre-main-sequence (PMS) stars having $U V$ excess. In Fig. 9 stars with $V \leq 16$ are shown by filled circles. The apparent distance modulus for NGC 663 is $\left(m-M_{V}\right)=14.4$ (cf. Pandey et al. 2002); the stars with $V=16$ (i.e. $M_{V}=1.6$ ) will

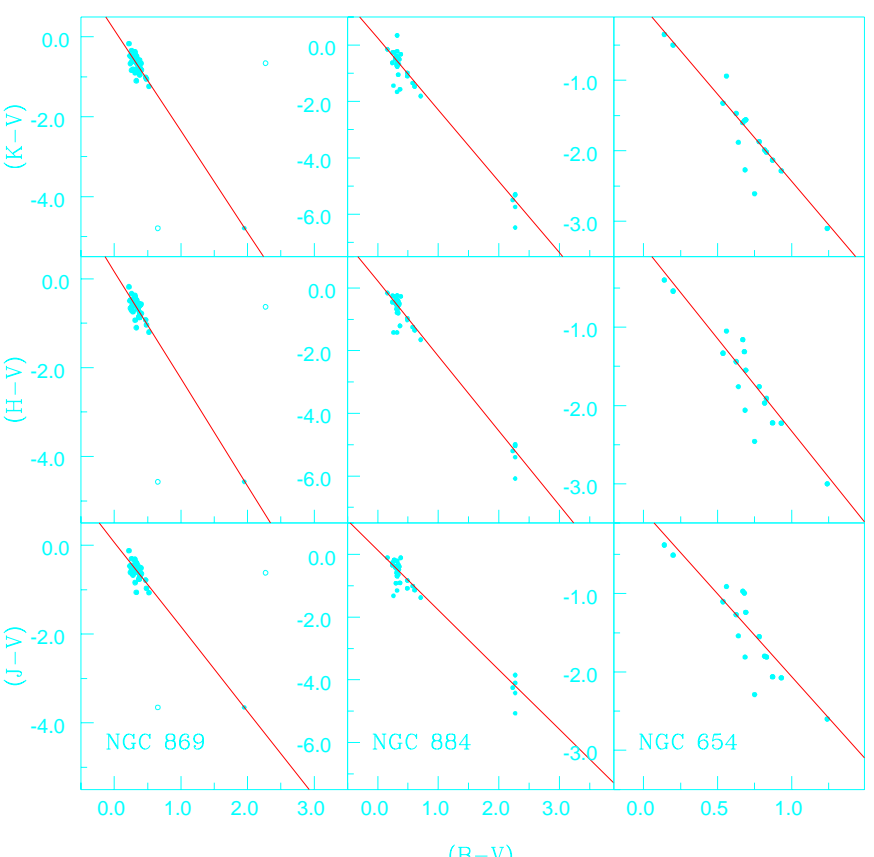

Fig. 8. Same as Fig. 7 but for the clusters NGC 869, NGC 884 and NGC 654.

have mass $\sim 2.5 M_{\odot}$. Since the cluster has an age of $\sim 10^{7} \mathrm{yr}$, the stars of $\sim 2.5 M_{\odot}$ should have reached the MS and no longer be PMS stars. The TCD for these stars also does not support a normal value of $X$. We find that the MS shifted along a reddening vector of 0.60 and $E(B-V)=0.68$ explains the observations satisfactorily.

The value of $X$ in the NGC 663 cluster region can further be checked by comparing the theoretical zero-age-main-sequence (ZAMS) with the observed stellar distribution in the $V /(B-V)$ and $V /(U-B)$ CMDs. Once the reddening is known from the $(U-B) /(B-V) \mathrm{CCD}$, the ZAMS is shifted to match the blue envelope of the observed stellar distribution in the $V /(B-V)$ and $V /(U-B)$ CMDs. The ZAMS fitting for $E(B-V)=0.68$ and apparent distance modulus $\left(m-M_{V}\right)=14.4$ is shown in Fig. 10a. Figure $10 \mathrm{~b}$ shows $V /(U-B)$ CMD where ZAMS, shifted for $E(U-B)=0.49$ (corresponding to the normal reddening vector $X=0.72)$ and $\left(m-M_{V}\right)=14.4$, is shown by a dashed line, which clearly shows disagreement with the distribution of the stars. The ZAMS for $E(U-B)=0.40$ (corresponding to $X=0.60$ and $E(B-V)=0.68)$ nicely fits the blue 


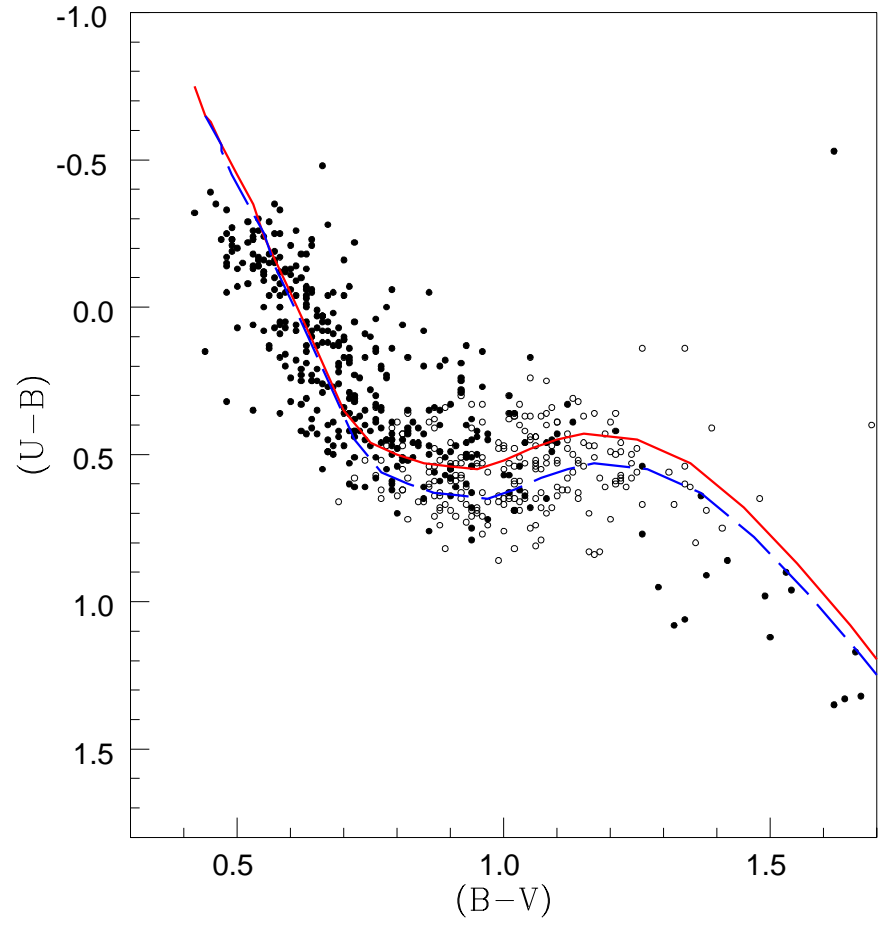

Fig. 9. $(U-B) /(B-V)$ two-colour diagram for the cluster NGC 663 . The continuous curve represents the MS shifted along $E(U-B) / E(B-$ $V)=0.60$, whereas dashed curve shows the MS shifted along a normal reddening vector. Star having $V \leq 16.0$ are shown by filled circles.

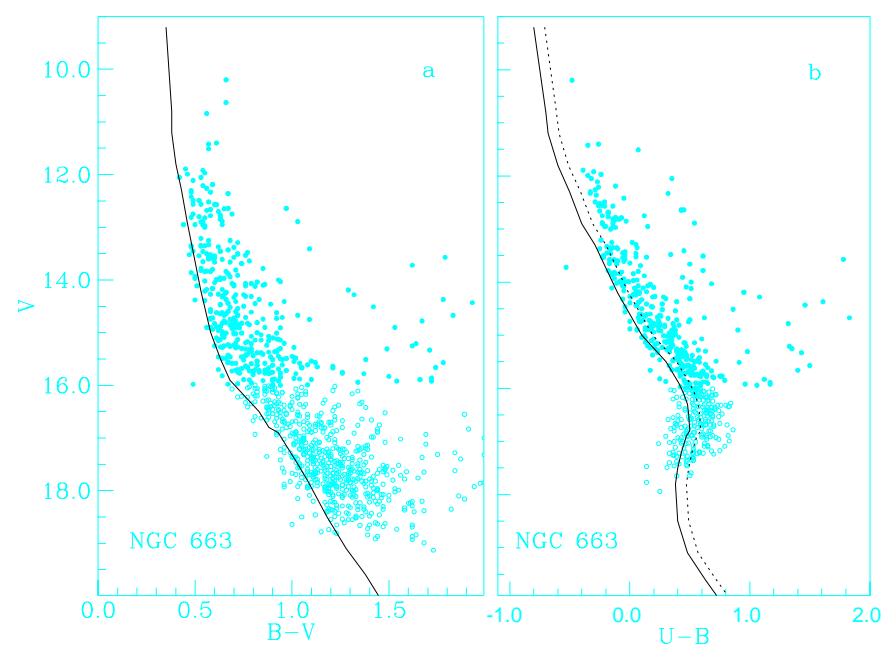

Fig. 10. $V / B-V$ (the left panel), $V / U-B$ (the right panel) CMDs for the cluster NGC 663. Star having $V \leq 16.0$ are shown by filled circles. The continuous curve in left panel represents the ZAMS fitting for $E(B-V)=0.68$ and apparent distance modulus $\left(m-M_{V}\right)=14.4$. The continuous and dashed curves in right panel shows ZAMS shifted for $E(U-B)=0.40$ and $E(U-B)=0.49$ respectively. For details see text.

envelope of the distribution. This supports an anomalous value $X=0.60$ for the slope in the NGC 663 cluster region.

$(U-B) /(B-V)$ TCDs and $V /(B-V)$ CMDs were used to find out the value of $X$ in all of the 14 clusters examined in the present study. The $(U-B) /(B-V)$ TCD for 3 clusters namely Be 62, NGC 436 and NGC 637 is shown in Fig. 11. The values of the reddening vector $X$ obtained from the TCD/CMDs are
Table 4. The value of the reddening vector $X=E(U-B) / E(B-V)$ obtained from the TCD/CMDs.

\begin{tabular}{ll}
\hline \hline Cluster & $X$ \\
\hline IC 1590 & 0.72 \\
Be 62 & 0.60 \\
NGC 436 & 0.84 \\
NGC 457 & 0.72 \\
NGC 581 & 0.72 \\
Tr 1 & 0.72 \\
NGC 637 & 0.53 \\
NGC 654 & 0.72 \\
NGC 663 & 0.60 \\
Be 7 & 0.72 \\
NGC 869 & 0.95 \\
IC 1805 & 0.72 \\
NGC 884 & 0.72 \\
NGC 1502 & 0.76 \\
\hline
\end{tabular}

given in Table 4. The uncertainty in the estimated value of $X$ arises due to uncertainties in the intrinsic colours (i.e. ZAMS), uncertainties associated with the observations and also uncertainties associated with the visual fit of the ZAMS to the observations. The typical total uncertainty in the reported values of $X$ is estimated to be $\sim 0.05$.

\section{The value of $R$}

Table 4 indicates that the clusters Be 62, NGC 637 and NGC 663 show a smaller value whereas the clusters NGC 869 and NGC 436 show a higher value for $X$. The remaining 8 clusters show a normal value for $X$. The data given in Tables 2 , 3 and 4 are used to estimate the weighted (according to associated errors) mean value of the colour excess ratios $E(\lambda-$ $V) / E(B-V)$ and the ratios are given in Table 5.

We define a parameter $r$ which is the ratio of $[E(\lambda-$ $V) / E(B-V)]_{\text {cluster }}$ (the ratio of colour excesses in the cluster region) and $[E(\lambda-V) / E(B-V)]_{\text {normal }}$ (the ratio of colour excesses for the normal reddening law). The values of $E(\lambda-V) / E(B-V)$ given in Table 5 are used to obtain the ratio $r$ for the cluster region and resultant value of $r$ is given in Table 6. For the clusters where the data from $U$ band to $K$ band are available, $r$ is plotted as a function of $\lambda^{-1}$ in Fig. 12.

Figure 12 indicates that the extinction in most of the cluster regions seems to be normal at $\lambda>\lambda_{I}$, except for the clusters NGC 1502 (where the colour excess ratios for $\lambda>\lambda_{I}$ are less than the normal one) and NGC 1805 (where the colour excess ratio at $\lambda \geq \lambda_{J}$ are higher than the normal one). Recent studies support a universality of the extinction curves for $\lambda>\lambda_{I}$ (see e.g. Cardelli et al. 1989; He et al. 1995). It is suggested that the normalization should be done using the $E(V-K)$ instead of $E(B-V)$ (Tapia et al. 1991) because the $E(V-K)$ does not depend on properties like chemical composition, shape, structure, degree of alignment of interstellar dust (cf. Mathis 1990 and references therein). 
Table 5. Weighted mean value of the colour excess ratios $E(\lambda-V) / E(B-V)$.

\begin{tabular}{lllllll}
\hline \hline Cluster & $\frac{E(U-V)}{E(B-V)}$ & $\frac{E(R-V)}{E(B-V)}$ & $\frac{E(I-V)}{E(B-V)}$ & $\frac{E(J-V)}{E(B-V)}$ & $\frac{E(H-V)}{E(B-V)}$ & $\frac{E(K-V)}{E(B-V)}$ \\
\hline NGC 654 & $1.72 \pm 0.07$ & $-0.65 \pm 0.03$ & $-1.35 \pm 0.07$ & $-2.18 \pm 0.25$ & $-2.42 \pm 0.24$ & $-2.60 \pm 0.26$ \\
NGC 663 & $1.60 \pm 0.07$ & $-0.55 \pm 0.02$ & $-1.28 \pm 0.04$ & $-2.43 \pm 0.29$ & $-2.84 \pm 0.33$ & $-3.08 \pm 0.36$ \\
NGC 869 & $1.95 \pm 0.07$ & $-0.64 \pm 0.03$ & $-1.28 \pm 0.02$ & $-2.16 \pm 0.16$ & $-2.54 \pm 0.15$ & $-2.65 \pm 0.17$ \\
NGC 884 & $1.72 \pm 0.07$ & $-0.66 \pm 0.03$ & $-1.21 \pm 0.02$ & $-2.29 \pm 0.09$ & $-2.61 \pm 0.09$ & $-2.77 \pm 0.11$ \\
NGC 1502 & $1.76 \pm 0.07$ & & & $-1.97 \pm 0.19$ & $-2.14 \pm 0.24$ & $-2.20 \pm 0.24$ \\
IC 1805 & $1.72 \pm 0.07$ & & $-1.25 \pm 0.05$ & $-2.55 \pm 0.22$ & $-2.86 \pm 0.23$ & $-3.07 \pm 0.24$ \\
Normal & 1.72 & -0.60 & -1.25 & -2.30 & -2.58 & -2.78 \\
\hline
\end{tabular}

Cardelli et al. (1989) found that the mean $R$ dependent extinction law can be represented by the following relation

$\frac{A_{\lambda}}{A_{V}}=a_{\lambda}+\frac{b_{\lambda}}{R}$

where $a_{\lambda}$ and $b_{\lambda}$ can be obtained from the relations given by Cardelli et al. (1989). The above relation can be written, in terms of $\frac{E(\lambda-V)}{E(B-V)}$, as

$\frac{E(\lambda-V)}{E(B-V)}=R\left(a_{\lambda}-1\right)+b_{\lambda}$.

The ratio of total-to-selective extinction towards the cluster direction " $R_{\text {cluster" }}$ " is derived using the Eq. (2). The value of $\frac{E(\lambda-V)}{E(B-V)}\left(\lambda \geq \lambda_{J}\right)$ given in Table 5 is used to estimate the value of $R_{\text {cluster }}$ and the results estimate for $R_{\text {cluster }}$ are given Table 6 . The clusters that have a broad spectrum of data are discussed below.

\section{NGC 654}

Sagar \& Yu (1989) concluded that at wavelengths greater than $5500 \AA$, the extinction is normal. The presence of unusually well aligned interstellar grains indicated by the polarization measurements seems to increase the extinction in the $U$ and $B$ bands slightly (Sagar \& Yu 1989). In the present work we find a rather normal extinction law. The value of $R_{\text {cluster }}$ is $\sim 2.97 \pm 0.30(\sigma)$ which, within the error, is close to the normal value of 3.1 .

\section{NGC 663}

Using the colour excesses $E(V-K)$ and $E(B-V)$ Tapia et al. (1991) found weak evidence for an anomalous reddening law with a value of $R_{\text {cluster }}=2.73 \pm 0.20$, which is marginally lower than the normal value of 3.1. However, they felt that the scatter in their data is too large to conclude about the value of $R_{\text {cluster }}$. Yadav \& Sagar (2001) reported values for $E(\lambda-V) / E(B-V)$ $\left(\lambda \geq \lambda_{J}\right)$ which are significantly smaller than the normal ones.

The (weighted) mean value of $R_{\text {cluster }}=3.50 \pm 0.40(\sigma)$ suggests a marginally anomalous reddening law in the NGC 663 cluster region but in the opposite sense to that reported by Tapia et al. (1991) and Yadav \& Sagar (2001). More near-IR data is needed to determine the $R_{\text {cluster }}$ in the NGC 663 cluster region. Here it is interesting to mention that the behaviour of the extinction curve towards UV also deviates from the normal one. Figure 12 indicates a lower value for the $E(U-V) / E(B-V)$ ratio, whereas Yadav \& Sagar (2001) reported a normal value for this ratio. They supplemented their data with the photometric spectral types which are based on the $Q$ method, where they adopted $E(U-B) / E(B-V)=0.72$. Presumably a dominance of photometric spectral determination forced the ratio of $E(U-B) / E(B-V)$ to a normal value.

\section{NGC 869 and 884 ( $h$ and $\chi$ Persei)}

From the extinction curve analysis Johnson (1965) found a value of $R_{\text {cluster }}=3.0$ in the NGC 869 and NGC 884 cluster region. Tapia et al. (1984) also reported a normal reddening law in the cluster region. Recently Yadav \& Sagar (2001) found that the $E(\lambda-V) / E(B-V)$ ratios for $\lambda \geq \lambda_{J}$ are smaller than the normal ones.

The colour excess diagrams (method "A") of NGC 869 indicates somewhat lower values for the ratios $E(J-V) / E(B-V)$ and $E(K-V) / E(B-V)$ but the errors are large, whereas in the case of NGC 884 the errors in the estimation of colour excess ratios are too large. The reason for the large errors is a small range in the $E(B-V)(\sim 0.3 \mathrm{mag})$. Because of the large errors we have not used the colour excess ratios obtained from the CEDs of NGC 869 in the further analysis. The TCDs (method "B") indicate that the ratio of colour excesses $E(\lambda-V) / E(B-V)$ in both the clusters for $\lambda \geq \lambda_{I}$ are perfectly normal. It is interesting to note that the CED for NGC 869 yields $E(U-V) / E(B-V)=$ $1.90 \pm 0.13$. The $V /(B-V)$ and $V /(U-B)$ CMDs also seem to support the ratio of $E(U-V) / E(B-V)=1.9$. In a recent study Keller et al. (2001) have adopted $E(U-B) / E(B-V)=0.72$ to fit the ZAMS to the stellar distribution on the $V /(U-B)$ CMD of the stars in the NGC 869 and NGC 884 cluster region. We find that only in the case of NGC 884 the $V /(U-B)$ CMD supports a normal value for the reddening vector $X$ in the cluster region.

\section{NGC 1502}

Tapia et al. (1991) found the ratio $E(V-K) / E(B-V)=2.20$ corresponding to $R_{\text {cluster }}=2.42 \pm 0.09$, which is significantly lower than the reddening value. Yadav \& Sagar (2001) also found that the colour excess ratios $E(\lambda-V) / E(B-V)$ for $\lambda \geq \lambda_{J}$ are significantly smaller than the normal ones. The colour excess ratios for $\lambda \geq \lambda_{J}$ obtained in the present study are in good agreement with those reported by Tapia et al. (1991) and Yadav \& Sagar (2001). The value of $R_{\text {cluster }}$ is estimated as $2.57 \pm 0.27$ which is in good agreement with that obtained by Tapia et al. (1991).

\section{IC 1805}

Various studies have been carried out to estimate the value of $R$ in the cluster region of IC 1805, but the results are not conclusive. Johnson (1968), Ishida (1969) and Kwon \& Lee (1983) 


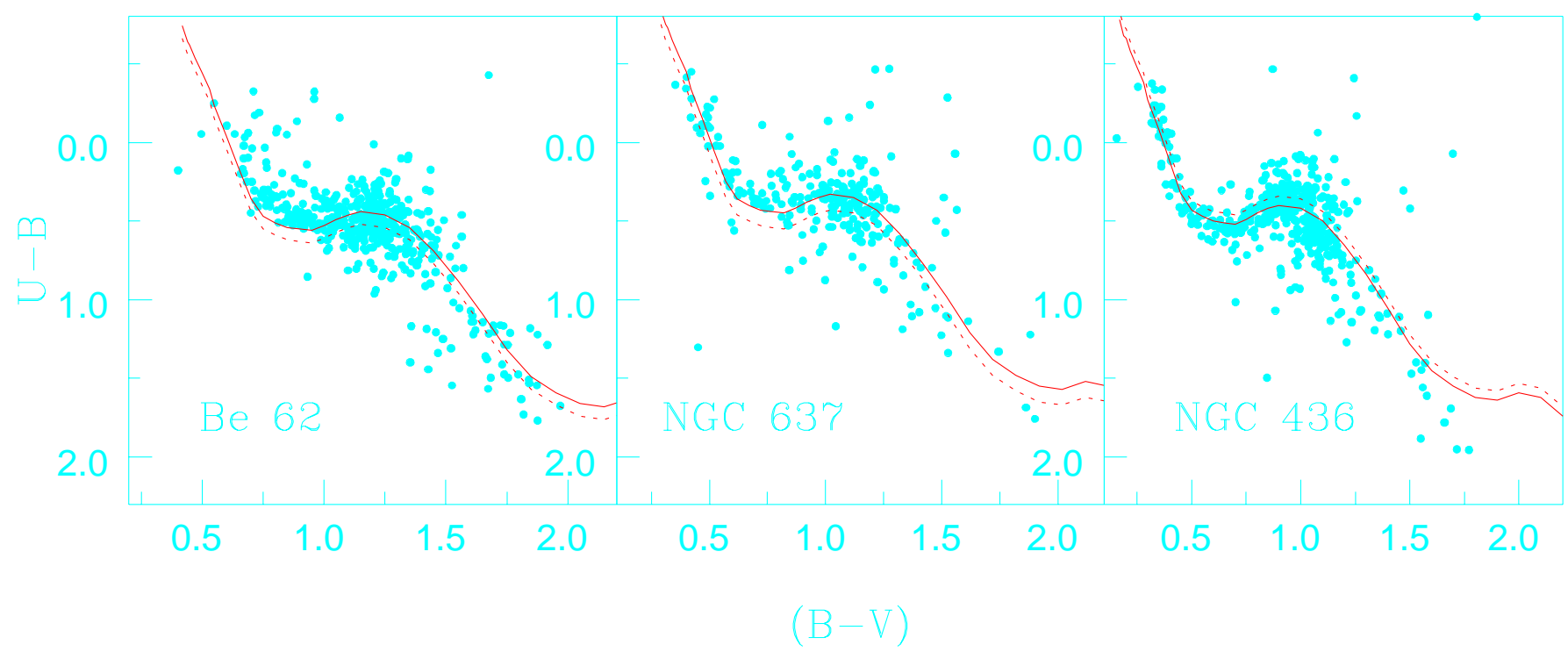

Fig. 11. CCDs for the clusters Be 62, NGC 436 and NGC 637. The continuous curve represents the MS shifted along the reddening vector given in Table 4 and dashed curve shows the MS shifted along a normal reddening vector.

Table 6. Mean value of the ratio $r$ as a function of wavelength.

\begin{tabular}{llllllll}
\hline \hline \multicolumn{1}{c}{$\lambda^{-1}\left(\mu \mathrm{m}^{-1}\right)$} & 2.90 & 1.56 & 1.25 & 0.80 & 0.61 & 0.45 & $R_{\text {cluster }}$ \\
Cluster & $U$ & $R_{\mathrm{C}}$ & $I_{\mathrm{C}}$ & $J$ & $H$ & $K$ & \\
\hline NGC 654 & $1.00 \pm 0.04$ & $1.08 \pm 0.05$ & $1.08 \pm 0.06$ & $1.07 \pm 0.14$ & $0.98 \pm 0.09$ & $0.95 \pm 0.03$ & $2.97 \pm 0.30$ \\
NGC 663 & $0.93 \pm 0.04$ & $0.92 \pm 0.04$ & $1.02 \pm 0.03$ & $1.07 \pm 0.11$ & $1.09 \pm 0.11$ & $1.10 \pm 0.11$ & $3.50 \pm 0.40$ \\
NGC 869 & $1.19 \pm 0.02$ & $1.07 \pm 0.05$ & $1.02 \pm 0.02$ & $0.98 \pm 0.05$ & $1.02 \pm 0.05$ & $1.00 \pm 0.04$ & $3.04 \pm 0.20$ \\
NGC 884 & $1.00 \pm 0.04$ & $1.02 \pm 0.05$ & $0.97 \pm 0.02$ & $0.97 \pm 0.03$ & $0.99 \pm 0.02$ & $0.98 \pm 0.03$ & $3.19 \pm 0.12$ \\
NGC 1502 & $1.02 \pm 0.03$ & & & $0.85 \pm 0.05$ & $0.83 \pm 0.05$ & $0.81 \pm 0.04$ & $2.57 \pm 0.27$ \\
IC 1805 & $1.00 \pm 0.03$ & & $1.00 \pm 0.02$ & $1.12 \pm 0.09$ & $1.14 \pm 0.08$ & $1.16 \pm 0.07$ & $3.56 \pm 0.29$ \\
\hline
\end{tabular}

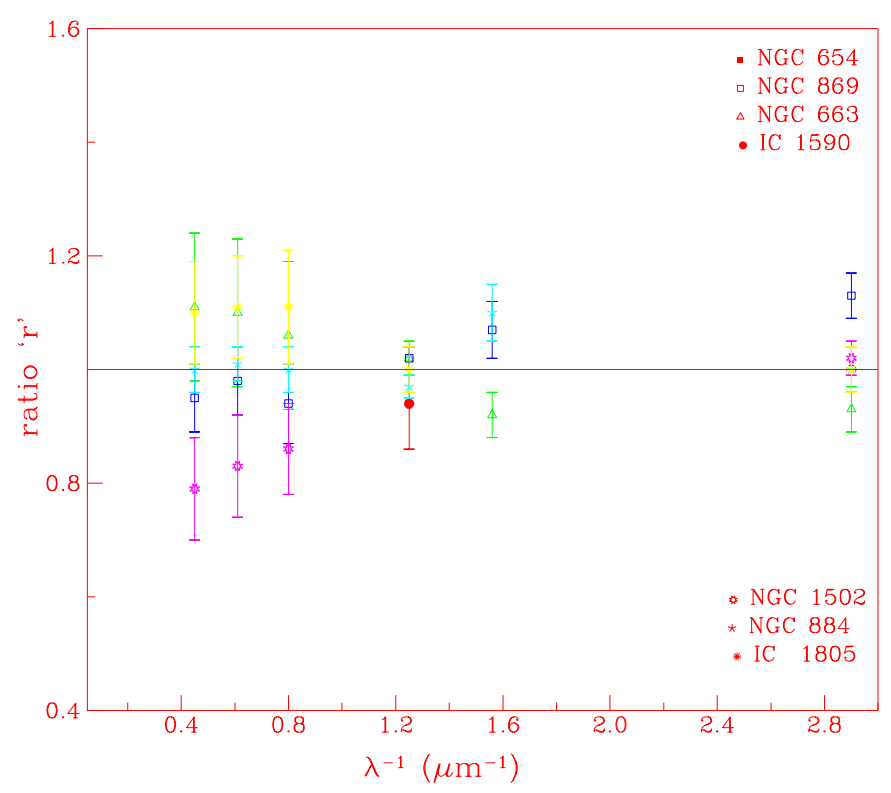

Fig. 12. The ratio $r$ as a function of $\lambda^{-1}$.

reported an anomalous reddening law in the cluster region with values of $R_{\text {cluster }} \sim 5.7, \sim 3.8$ and $\sim 3.44$ respectively. Kwon $\&$ Lee also reported a regional variation in the value of $R_{\text {cluster }}$, with a maximum value of $R_{\text {cluster }}=3.82 \pm 0.15$ for stars located in the outer region and the minimum of $3.06 \pm 0.05$ for stars located in the central region. Sagar \& Yu (1990) found that the interstellar extinction law in the direction of most of the cluster members is normal. The colour excess ratios $E(\lambda-V) / E(B-V)$ for $\lambda \geq \lambda_{J}$ obtained in the present work indicate an anomalous reddening law in the cluster region of IC 1805. The value of $R_{\text {cluster }}$ is estimated as $3.56 \pm 0.29$, which also indicates an anomalous reddening behaviour in the cluster region.

\section{The $A_{\lambda} / A_{\mathrm{V}}$ curve}

Extinction has normally been analyzed using a two-colour normalization of the form $E(\lambda-V) / E(B-V)$. However, the true nature of the variability of observed extinction may be hidden by the choice of normalization. The quantity $A_{\lambda} / A_{V}$ reflects a more fundamental extinction behaviour than the $E(\lambda-V) / E(B-V)$ (cf. Cardelli et al. 1989). The average colour excess ratios given in Table 5 can be used to estimate the quantity $A_{\lambda} / A_{V}$ in the following manner,

$A_{\lambda} / A_{V}=\left[E(\lambda-V) / E(B-V) R_{\text {cluster }}\right]+1$

where $R_{\text {cluster }}$ is taken from Table 6. In Fig. 13a the normalized extinction in the form $A_{\lambda} / A_{V}$ is plotted against $\lambda^{-1}$ for the clusters NGC 654, NGC 663, NGC 869 and NGC 884 alongwith 
the average extinction law for $R_{\text {cluster }}=3.1$ given by Cardelli et al. (1989). Figure 13a indicates that the agreement between observations and the extinction law by Cardelli et al. (1989) is good barring the $A_{U} / A_{V}$ values for NGC 869 and NGC 663. In the case of NGC 869 the ratio $A_{U} / A_{V}$ is higher than the normal one whereas in the case of NGC 663 it is lower than the normal one. The value of $A_{U} / A_{V}=1.46$ in the case of NGC 663 supports $R_{\text {cluster }} \sim 3.5$. Whereas in the case of NGC 869 the colour excess ratios indicate a perfectly normal reddening law. It seems somewhat strange that in the case of these two clusters the ISM behaves at $\lambda_{U}$ in a different way.

The extinction law in the direction of two clusters IC 1502 and IC 1805 is found to be anomalous. The normalized extinction $A_{\lambda} / A_{V}$ for these two clusters along with the $\rho$ Oph dark cloud (data taken from Martin \& Whittet 1990) is plotted in Fig. $13 \mathrm{~b}$. The effect of varying $R_{\text {cluster }}$ on the shape of the extinction curves is quite apparent at the shorter wavelengths for different environments of the star forming regions.

As we have discussed the extinction in the direction of star clusters arises due to the general ISM in the foreground of the cluster and also due to the cloud associated with the cluster. Various studies using OB type single stars support a value of $R \sim 3.1$ for the general ISM (Wegner 1993; Lida et al. 1995; Winkler 1997). The minimum reddening, $E(B-V)_{\min }$, towards the direction of the cluster is representative of reddening due to the foreground dust. The slopes of the distribution of stars having $E(B-V) \leq E(B-V)_{\min }$ on the TCDs can give information about the foreground reddening law. In the case of IC 1805 (and NGC 654), where $E(B-V)_{\min }$ is $0.65(0.77)$, we used stars having $E(B-V) \leq 0.80(0.85)$ to estimate the foreground reddening presuming that star having $0.65(0.77) \leq E(B-V) \leq 0.80(0.85)$ are not much affected by the anomalous reddening law in the cluster region. The colour excess ratios $E(J-V) / E(B-V)$, $E(H-V) / E(B-V)$, and $E(K-V) / E(B-V)$ obtained are $-2.00 \pm 0.32(-1.98 \pm 0.28),-2.58 \pm 0.35(-2.47 \pm 0.27)$ and $-2.80 \pm 0.39(-2.82 \pm 0.36)$ respectively, which support a normal reddening law in front of the cluster IC 1805. We further combined data of all the clusters having stars with reddening $E(B-V) \leq 0.50$. We feel that the limit of $E(B-V) \leq 0.50$ safely excludes the reddening due to intra-cluster matter as the smallest $E(B-V)$ for the NGC 869 and NGC 884 is $\approx 0.50$ (e.g. Uribe et al. 2002). A least-squares fit to 8 data points having $0.01 \leq E(B-V) \leq 0.50$ gives the colour excess ratio $E(J-V) / E(B-V)=-2.23 \pm 0.32, E(H-V) / E(B-V)=$ $-2.62 \pm 0.22$, and $E(K-V) / E(B-V)=-2.79 \pm 0.26$. These colour excess ratios also indicate a normal foreground reddening law towards the direction of the clusters used in the present study.

Several studies have pointed to the apparent concentration of stars with high $R$-values in the vicinity of star forming regions. This effect has, for example, in the $\eta$ Carina nebula (Forte 1978; Thé \& Groot 1983), M 16 (Chini \& Wargau 1990) and M 17 (Chini et al. 1980; Chini \& Wargau 1998) and it may be presumed to be characterstic of many more HII regions (Winkler 1997). Winkler (1997) compared the value of $R$ obtained for hottest stars in the Galaxy (spectral type O8 or earlier), which can be considered as indicators of regions with recent star formation, and he confirms that in the majority of the

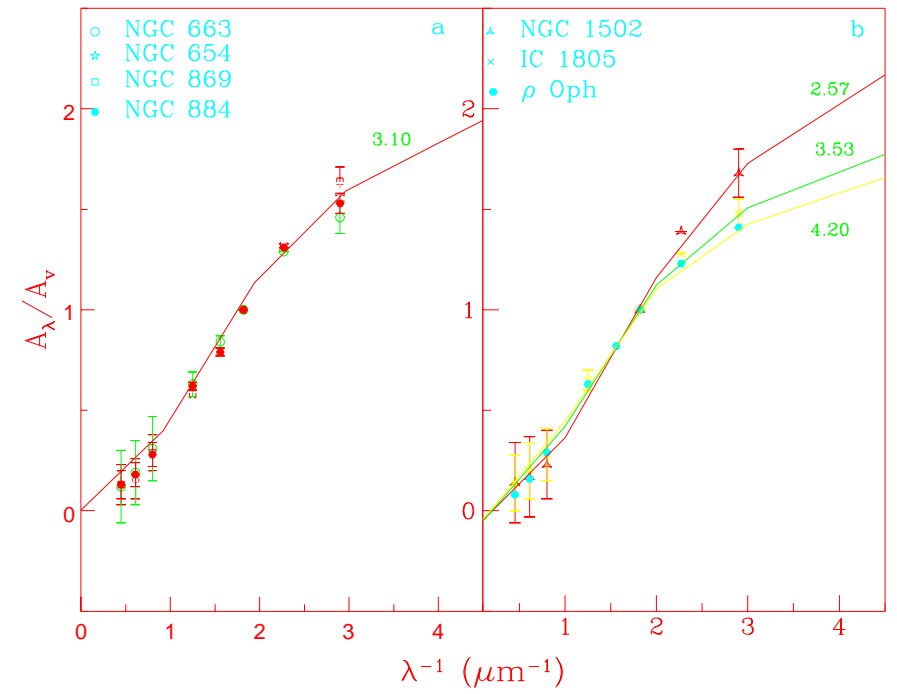

Fig. 13. The normalized extinction curves $A_{\lambda} / A_{V}$. The values written along the curves represent the value of $R_{\text {cluster }}$.

cases the stars with large $R$ indeed seems to be in the vicinity of star forming regions.

On the basis of the above discussions we presume that the anomalous extinction law in the direction of cluster IC 1805 is due to the intra-cluster material.

\section{Conclusions}

In the present work we have carried out a detailed study of the intra-cluster material inside the young open clusters within a range of galactic longitude $123^{\circ}<l<144^{\circ}$. We used three methods (cf. Sect. 3.1) to derive $E(\lambda-V) / E(B-V)$ and the results agree with each other in a few percent. It is found that the behaviour of extinction varies from cluster to cluster. The main results are as follows.

1. The extinction curves at shorter wavelengths depend upon the $R_{\text {cluster }}$ while they converge for $\lambda \geq \lambda_{J}$.

2. The extinction behaviour in the case of NGC 654, NGC 869 and NGC 884 is found normal, whereas in the case of NGC 663 there is some tendency for a higher value of $R_{\text {cluster. }}$ It is interesting to mention that in the case of NGC 663 and NGC 869 the extinction at $\lambda_{U}$ is found different from the normal one. In the case of NGC 663 the extinction process in the $U$ band seems to be less efficient, whereas in the case of NGC 869 the process is more efficient.

3. The cluster regions of IC 1502 and IC 1805 show anomalous reddening laws. In the case of IC 1502 the $R_{\text {cluster }} \sim$ $2.57 \pm 0.16$ which is in agreement with the value $(\sim 2.49 \pm$ 0.09 ) obtained by Tapia et al. (1991), whereas in the case of IC 1805 the present study yields $R_{\text {cluster }} \sim 3.53 \pm 0.25$ which is in agreement with the value $(\sim 3.44)$ given by Kwon \& Lee (1983).

More near-IR data is needed for the clusters NGC 663, NGC 1502 and IC 1805, where an anomalous value of $R_{\text {cluster }}$ is found, to study the reddening law in detail in the these clusters. 
Acknowledgements. This work is partly supported by the DST (India) and the JSPS (Japan). AKP is thankful to the staff of KISO observatory for their help during his stay there. Authors are grateful to Prof. Ram Sagar for useful discussions. Thanks are also due to Dr. John Mathis for his comments which improved the contents of the paper.

\section{References}

Bessel, M. S. 1979, PASP, 91, 589

Cardelli, J. A., Clayton, G. C., \& Mathis, J. S. 1989, ApJ, 345, 245

Chini, R., Elsässer, H., \& Neckel, T. 1980, A\&A, 91, 186

Chini, R., \& Wargau, W. F. 1990, A\&A, 227, 213

Chini, R., \& Wargau, W. F. 1998, A\&A, 329, 161

DeGioia Eastwood, K., Throop, H., Walker, G., \& Cudworth, K. M. 2001, ApJ, 549, 578

Elmegreen, B. G., \& Lada, C. J. 1977, ApJ, 214, 725

Fitzpatrick, E. L. PASP, 1999, 111, 63

Forte, J. C. 1978, AJ, 83, 1199

He, L., Whittet, D. C. B., Kilkenny, D., \& Spencer Jones, J. H. 1995, ApJS, 101, 335

Johnson, H. L. 1965, ApJ, 141, 923

Johnson, H. L. 1966, ARA\&A, 4, 193

Johnson, H. L. 1968, in Nebulae and Interstellar Matter, ed. B. M. Middlehurst, \& L. M. Aller (University of Chicago Press), 5

Ishida, K. 1969, MNRAS, 144, 55

Kiszkurno, E., Kołos, R., Krełowski, J., \& Strobel, A. 1984, A\&A, 135,337

Koornneef, J. 1983, A\&A, 128, 84
Krełowaski, J., \& Strobel, A. 1983, A\&A, 127, 271

Krełowaski, J., \& Strobel, A. 1987, A\&A, 175, 186

Kwon, S. M., \& Lee, S. W. 1983, JKAS, 16, 7

Lida, H., Whittet, D. C. B., Kilkenny, D., \& Spencer Jones, J. H. 1995, ApJS, 101, 335

Mathis, J. S. 1990, ARA\&A, 28, 37

Martin, P. G., \& Whittet, D. C. B. 1990, ApJ, 357, 113

Mermilliod, J. C. 1995, in Information \& On-line Data in Astronomy, ed. D. Egret, \& M. A. Abrecht (Kluwer Academic Press), 227

Pandey, A. K., Mahra, H. S., \& Sagar, R. 1990, AJ, 99, 617

Pandey, A. K., Ogura, K., \& Sekiguchi, K. 2000, PASJ, 52, 847

Pandey, A. K., Upadhyay, K., \& Ogura, K., et al. 2002, in preparation

Phelps, R. L., \& Janes, K. A. 1994, ApJS, 90, 31

Sagar, R., \& Yu, Q. Z. 1989, MNRAS, 240, 551

Schmidt-Kaler, Th. 1982, in Landolt-Börnstein, Neue Serie Gr. VI, vol. 2b (Springer, Berlin, Heidelberg, New York), 19

Tapia, M., Roth, M., Costero, R., \& Navarro, S. 1984, Rev. Mex. Astron. Astrofís., 9, 65

Tapia, M., Costero, R., Echevarria, J., \& Roth, M. 1991, MNRAS, 253, 649

Thé, P. S., \& Groot, M. 1983, A\&A, 125, 75

Turner, D. G. 1994, Rev. Mex. Astron. Astrofís., 29, 163

Uribe, A., García-Varela, J., Sabogal-Martínez, B., et al. 2002, PASP, 114,233

Wegner, W. 1993, AcA, 43, 209

Winkler, H. 1997, MNRAS, 287, 481

Whittet, D. C. B. 1977, MNRAS, 180, 29

Yadav, R. K. S., \& Sagar, R. 2001, MNRAS, 328, 370 